\title{
Radio spectroscopy of stellar flares: magnetic reconnection \& CME shocks in stellar coronae
}

\author{
Jackie Villadsen $^{1}$, Gregg Hallinan ${ }^{1}$ and Stephen Bourke ${ }^{1}$ \\ ${ }^{1}$ Department of Astronomy, California Institute of Technology \\ MC 249-17, 1200 E California Blvd, Pasadena, CA 91125, USA \\ email: jrv@astro.caltech.edu, gh@astro.caltech.edu, sb@astro.caltech.edu
}

\begin{abstract}
High-cadence spectroscopy of solar and stellar coherent radio bursts is a powerful diagnostic tool to study coronal conditions during magnetic reconnection in flares and to detect coronal mass ejections (CMEs). We present results from wide-bandwidth VLA observations of nearby active $\mathrm{M}$ dwarfs, including some observations with simultaneous VLBA imaging. We also discuss the Starburst program, which will make wide-bandwidth radio spectroscopic observations of nearby active flare stars for $20+$ hours a day for multiple years, coming online in spring 2016 at the Owens Valley Radio Observatory. This program should vastly increase the diversity of observed stellar radio bursts and our understanding of their origins, and offers the potential to detect a population of CME-associated radio bursts.
\end{abstract}

Keywords. stars: coronae, stars: flare, stars: individual (UV Ceti), stars: mass loss, Sun: coronal mass ejections (CMEs), Sun: radio radiation, radio continuum: stars

\section{Introduction}

Many of the nearest habitable-zone Earths and super-Earths will be found around M dwarfs (Dressing \& Charbonneau 2013). The magnetically active "youth" of M dwarfs lasts hundreds of millions to billions of years (West et al. 2008). Active M dwarfs' high flare rates imply that coronal mass ejections (CMEs) may dominate their mass loss (Drake et al. 2013, Osten \& Wolk 2015). CMEs from active M dwarfs are predicted to cause significant mass loss from planetary atmospheres (Khodachenko et al. 2007, Lammer et al. 2007) and to accelerate energetic protons that drive drastic transient changes in molecular chemistry of planetary atmospheres, including the biomarker ozone (Segura et al. 2010). However, these theoretical predictions extrapolate from solar phenomena by orders of magnitude in energy into stellar environments with magnetic topologies very different from the Sun's. This extrapolation is necessary because there are currently no observations of CMEs on stars other than the Sun. Dynamic spectroscopy of stellar radio bursts offers the potential to detect these unseen stellar CMEs.

The Sun produces a diversity of coherent radio bursts, with distinct patterns in the time-frequency plane depending on the mechanism producing them (such as CME shock fronts or magnetic reconnection in flares). The coherent nature of the emission is indicated by high brightness temperatures, short-timescale variability, narrow bandwidth, and/or strong circular polarization. Coherent emission occurs when electrons with an inverted velocity distribution (more high-velocity than low-velocity electrons) excite unstable wave growth, enabling brightness temperatures much higher than the local plasma temperatures. For solar radio bursts, these electrons may be accelerated at magnetic reconnection sites or by scattering off of CME shock fronts. For radio pulses from planets and brown dwarfs, the electrons can be supplied by magnetic interaction with the solar 
wind or with a satellite. The coherent emission mechanism (reviewed in Dulk 1985) may be plasma emission $\left(\nu \propto n_{e}^{1 / 2}\right)$, which is responsible for CME-associated radio bursts, or electron cyclotron maser emission $(\nu \propto B)$. The radiation is generally at the first or second harmonic, with the strongest circular polarization seen at the first harmonic.

Since the plasma frequency depends on the local plasma density, the evolution of a radio burst's emission frequency over time (the frequency drift rate) can be used to measure the source velocity across a density gradient. Solar CME shock fronts, with speeds of order $1000 \mathrm{~km} / \mathrm{s}$, produce radio bursts that sweep downwards through a factor of a few in frequency over the course of minutes. Electron beams, with speeds of order $0.1 c$, produce short-duration bursts with much more rapid frequency drift. Since coherent radio bursts can be intensely bright, they offer the potential to detect these processes in the coronae of nearby stars.

Dynamic spectroscopy (examining spectral evolution over time) of coherent radio bursts has provided evidence of coronal plasma motion on active $\mathrm{M}$ dwarfs (Bastian \& Bookbinder 1987; Osten \& Bastian 2006, 2008). Observations of stellar coherent radio bursts have, until recently, been limited by the fractional bandwidth $\left(\Delta \nu / \nu_{0}<1\right.$, where $\nu_{0}$ is the lower edge of the band). Recent upgrades to radio facilities, especially the Karl G. Jansky Very Large Array (VLA)'s WIDAR correlator and subarray capabilities, now offer the sensitivity and the large fractional bandwidth $\left(\Delta \nu / \nu_{0}>1\right)$ needed to track sources across multiple coronal scale heights, which helps to identify the mechanism producing the bursts (such as CME shock fronts).

\section{VLA wideband dynamic spectroscopy of active $M$ dwarfs}

In 2011, observations of UV Ceti with the VLA's new S band (2-4 GHz) receivers serendipitously caught a bright coherent burst (Hallinan et al. in prep). This burst, a few minutes in duration, sweeps slowly upwards in frequency, possibly indicating a plasma disturbance propagating downwards towards the star (into higher coronal densities). 2013 VLA observations used the VLA's subarray capabilities to observe 1-6 GHz simultaneously, detecting two bright coherent bursts on UV Ceti, with complex burst morphology suggestive of multiple reconnection sites.

To search for lower-frequency emission from an outward-propagating disturbance such as a CME, in 2015 we conducted VLA observations of five active M dwarfs using subarrays to observe 230-490 MHz and 1-4 GHz. In three 4-hour observations of UV Ceti we detected three long-duration bursts (the 1-4 GHz spectrum for the first of these is shown in Figure 1) and two short-duration bursts. The coherent burst in Figure 1 is detected from $250 \mathrm{MHz}$ to $8.5 \mathrm{GHz}$ (with the VLBA; see below), spanning a factor of $>30$ in frequency. The high degree of circular polarization of the burst (50-100\% at different frequencies) indicates emission at the fundamental frequency; for cyclotron maser emission this implies field strengths from 130 to $3000 \mathrm{G}$, and for plasma emission, densities from $10^{9}$ to $10^{12}$ $\mathrm{cm}^{-3}$. Some features with positive frequency drift, and some negative, are seen in the burst, implying source motion both towards and away from the star.

All of the UV Ceti coherent bursts detected in 2011, 2013, and 2015 show consistent strong right circular polarization. Since the orientation of the magnetic field determines the circular polarization, this consistency indicates that a persistent magnetic structure may be responsible for all of the bursts.

An upcoming paper (Villadsen et al. in prep) will present an analysis of the frequency drift rates observed in the dynamic spectra of the UV Ceti bursts from 2013 and 2015, including 230-490 MHz; these lower frequencies are especially significant for searching for an outward-propagating disturbance such as a CME. The paper will include observations 


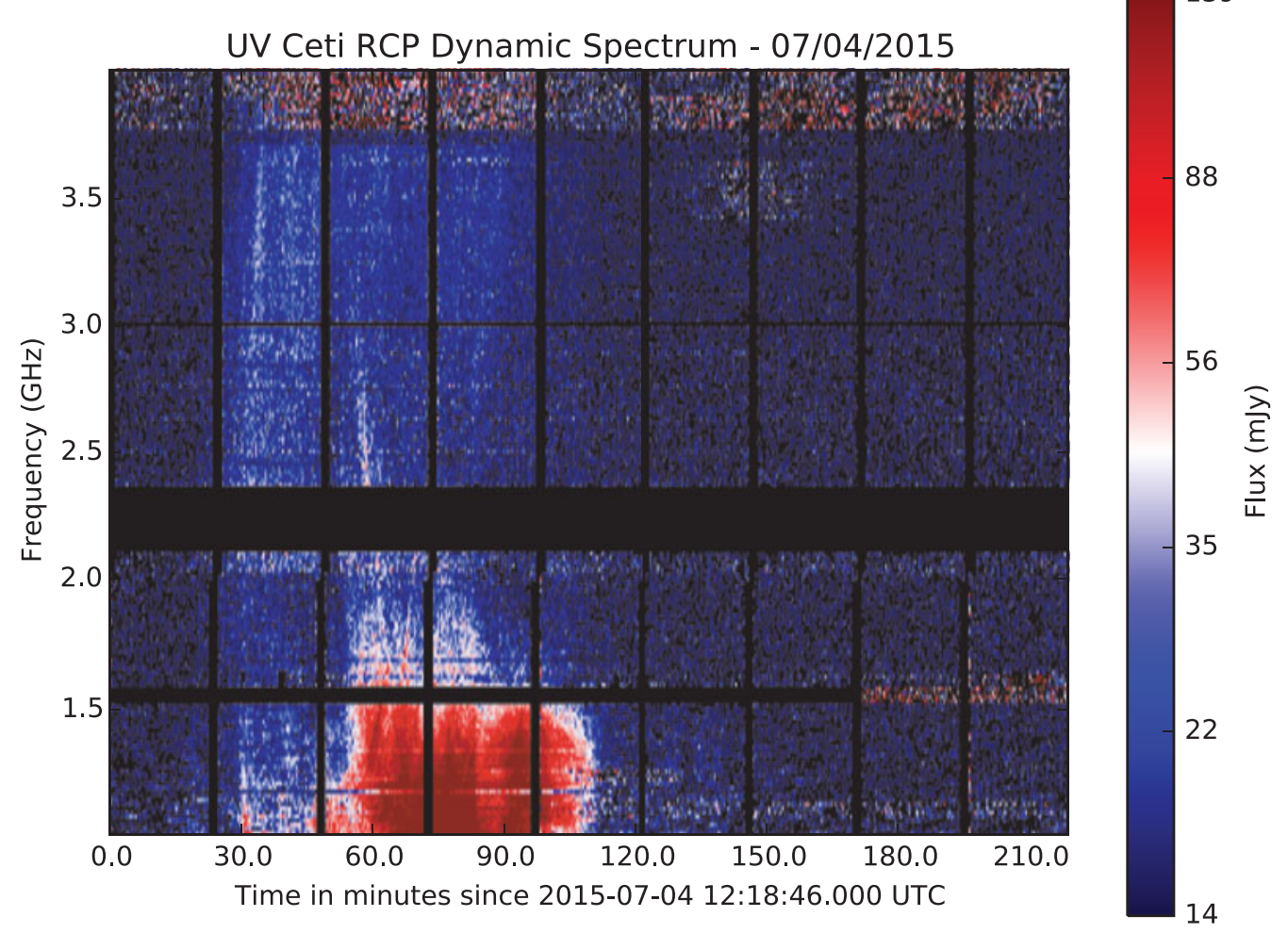

Figure 1. Dynamic spectrum of the right circularly polarized emission from an 80-minute-long coherent radio burst on UV Ceti. The burst has uniform circular polarization of about $70 \%$.

of four additional active $\mathrm{M}$ dwarfs, and present simultaneous Very Long Baseline Array 8.2-8.5 GHz images of the corona.

\subsection{Interpretation}

There are multiple scenarios relevant to interpreting the observed UV Ceti radio bursts:

- Downwards-moving shock front. The 2011 UV Ceti burst drifts slowly to higher frequencies, much too slowly to be attributed to an electron beam. To determine whether the emission might instead be attributed to a downwards-propagating shock front, the inferred source speed can be compared to the estimated local Alfvén speed.

- Multiple/moving reconnection sites. Emerging magnetic flux or complex flare morphology can result in multiple reconnection sites, which may account for bursts of emission at different sites such as seen in the dynamic spectrum of a 2013 burst on UV Ceti.

- Absorption by chromospheric evaporation front. A chromospheric evaporation front during a flare may cause a high-frequency cutoff for plasma emission due to free-free absorption by the evaporation front (Aschwanden \& Benz 1995), with the cutoff frequency decreasing over time as the evaporation front rises into regions with lower ambient densities. However, note that the timescale for this process in solar bursts is much more rapid (timescale of seconds) than the tens-of-minutes burst in Figure 1.

- Rotationally modulated auroral emission. The consistent direction and degree of circular polarization of the bursts from 2011-2015 suggests that the emission may be rotationally modulated like radio aurorae on ultracool dwarfs (Hallinan et al. 2006) and 
solar system planets. In aurorae, burst duration and frequency drift can be a geometric effect caused by rotation, rather than indicating actual source motion. If the observed bursts are rotationally modulated, we should see recurring bursts each rotation period, which UV Ceti's $v \sin i$ (Jones et al. 2005) implies is $<6$ hours. Repeated long-duration observations of UV Ceti on successive days will quickly resolve this question.

Lower-frequency data and very long baseline imaging, to be presented in the upcoming publication, will help differentiate among these scenarios, as will upcoming observations of UV Ceti over multiple rotation periods (which would immediately identify periodic auroral emission) planned as part of the Starburst program.

\section{The Starburst program: A dedicated radio facility for stellar dynamic spectroscopy}

The Starburst program is a new radio facility for dynamic spectroscopy of stellar radio bursts, coming online in spring 2016. Starburst consists of a 1-6 GHz single-baseline interferometric backend (Monroe et al. in prep) for the two 27-m telescopes at the Owens Valley Radio Observatory. The Owens Valley Solar Array (OVSA) is refurbishing these antennas with cooled receivers $\left(T_{s y s} \sim 50 \mathrm{~K}\right)$ for calibrating small solar-observing antennas. Outside of periodic solar calibration observations, the system will conduct stellar observations for many hours a day, with $10 \mathrm{mJy}$ rms sensitivity for dynamic spectrum pixels of $15 \mathrm{sec}$ and $20 \mathrm{MHz}$.

The Starburst program will monitor nearby active $\mathrm{M}$ dwarfs (and other active stars) to search for the signature of CME shock fronts, with simultaneous observations with a small optical telescope. An automatic burst detection pipeline for the Starburst radio dynamic spectra will be used to generate triggers for the VLBA in order to image the flaring radio corona, which is sometimes resolved for active $\mathrm{M}$ dwarfs (Benz et al. 1998). UV Ceti is one of the planned Starburst targets; these observations will resolve the question of whether frequency sweep in UV Ceti dynamic spectra is due to rotational modulation of auroral radio emission or whether it traces motion of coronal plasma.

\section{Acknowledgements}

This material is based upon work supported by the National Science Foundation under Grant No. AST-1311098. The National Radio Astronomy Observatory is a facility of the National Science Foundation operated under cooperative agreement by Associated Universities, Inc. Student travel to present this talk was funded by the SETI Institute and the NASA Astrobiology Institute.

\section{References}

Aschwanden, M. J. \& Benz, A. O. 1995, ApJ, 438, 997

Bastian, T. S. \& Bookbinder, J. A. 1987, Nature, 326, 678

Benz, A. O., Conway, J., \& Gudel, M. 1998, A\& A, 331, 596

Drake, J. J., Cohen, O., Yashiro, S., \& Gopalswamy, N. 2013, ApJ, 764, 170

Dressing, C. D. \& Charbonneau, D. 2013, ApJ, 767, 95

Dulk, G. A. 1985, ARAA, 23, 169

Hallinan, G., Antonova, A., Doyle, J. G., Bourke, S., Brisken, W. F., \& Golden, A. 2006, ApJ, 653,690

Jones, H. R. A., Pavlenko, Y., Viti, S., Barber, R. J., Yakovina, L. A., Pinfield, D., \& Tennyson, J. 2005, MNRAS, 358, 105

Khodachenko, M. L., Ribas, I., Lammer, H., Grießmeier, J.-M., Leitner, M., Selsis, F., Eiroa, C., Hanslmeier, A., Biernat, H. K., Farrugia, C. J., \& Rucker, H. O. 2007, Astrobiology, 7, 167 
Lammer, H., Lichtenegger, H. I. M., Kulikov, Y. N., Grießmeier, J.-M., Terada, N., Erkaev, N. V., Biernat, H. K., Khodachenko, M. L., Ribas, I., Penz, T., \& Selsis, F. 2007, Astrobiology, 7, 185

Osten, R. A. \& Bastian, T. S. 2006, ApJ, 637, 1016

Osten, R. A. \& Bastian, T. S. 2008, ApJ, 674, 1078

Osten, R. A. \& Wolk, S. J. 2015, ApJ, 809, 79

Segura, A., Walkowicz, L. M., Meadows, V., Kasting, J., \& Hawley, S. 2010, Astrobiology, 10, 751

West, A. A., Hawley, S. L., Bochanski, J. J., Covey, K. R., Reid, I. N., Dhital, S., Hilton, E. J., \& Masuda, M. 2008, AJ, 135, 785 